\title{
The Design Concept and Realization of University Library's Online Lecture System
}

\author{
Linbo Duan ${ }^{1,}$, ,Ping Qin ${ }^{1}$,Lingfei Qian ${ }^{1}$, Ting Xie ${ }^{1}$, Qingfeng Duan ${ }^{2}$ \\ ${ }^{1}$ Library,Nanjing University of Aeronautics and Astronautics,Nanjing, China \\ ${ }^{2}$ State Grid Corporation of China,Luohe power supply company,Luohe,China
}

\begin{abstract}
The traditional library lectures are based on the physical space environment, which is problematic in terms of user needs analysis, time selection, and resource utilization, so that the value of the library unexploited.University library's online lecture system adopts the three-tier architecture design pattern, which based on ASP.NET as the development platform and SQL Server as the back-end database, designing the system to solve the problems in the traditional library service.The design and implementation of the University library's online lecture system broke the traditional library lecture mode, and further optimized the library service concept, improve the quality of service to meet the diverse needs of the user groups. Integrating online test, interactive question and answer, feedback mechanism to the system, the system has a good user experience, visual way to show the audience's learning situation and other information, optimize the content and quality of services to make comments and suggestions.
\end{abstract}

\section{INTRODUCTION}

University libraries as the literature information centers and resource sharing platforms, play an important role in teaching and scientific research.A series of lectures carried out by university libraries are important parts of college education and teaching. At the same time these lectures also play an important role in the aspect of educational functions. The traditional library services mainly include the construction of information retrieval work, retrieval of documents and databases ,manual retrieval of documents and so on.The digital library pay more attention to service mobility and flexibility, and they focus on the development of disciplines in colleges and universities with the application of network and information technology. The duties and responsibilities of university libraries are carrying out service work about literature and information according to different user groups .In the form of giving lectures in libraries, making user groups have a thorough understanding of the library resources present situation and the application method, and learn more about literature delivery, better serving the teaching and scientific research.

The traditional library service model has been unable to meet the diverse needs of the user groups, and the informational lecture mode is expected to change the current situation.Professor Su Xinning at Nanjing University point out in the article [1] "large data age digital library facing opportunities and challenges"that:Future digital library want to jump out of the traditional framework for resource development, turn the passive wait into a positive activity, establish a comprehensive service concept and service mode and the form of resource dissemination. digital librarians should be determined to become data analysts.Wu Weici, professor at Peking University points out[2], in the sixth session of the Chinese Library Association report that:The development of modern library should use the new information technology as much as possible, and make the library development towards the direction of network and information.In the "Library Development Trend Research Report: Environmental Analysis and Main Strategy", Chu Jingli and Wu Dongman pointed out that:Library development should change, take the initiative to respond, analyze user needs and environmental changes.Research and develop a sustained and dynamic development strategy.Therefore, in the ever-changing external environment, Service concept and service form of libraries should also change with the external environment changes.

\section{The Necessity of Systematic Design of University Library 's Online Lecture}

According to the different needs of different user groups for library resources Construction and development of modern digital library.The design and implementation of online lecture system in University Libraries will solve some problems in the process of Library Construction. At the same time,the function of online evaluation of the system will contribute to perfecting the function of University Library,improving service quality,enhancing user experience,further enhancing the information literacy of user groups, and realizing the extension and expansion of library service mode and service scope in the "Internet plus" era. 


\subsection{Meet multi-level needs and make value of lectures Survivability Structure Model of Large-scale Network}

The service tenet of the library must be people-oriented,and the service form is bound to develop in the direction of diversification and hierarchy.The library user groups are characterized by diversity.Based on the teachers and students in the school and the related scientific research personnel, there are some differences between the different user groups in the demand level of the University library.Liang Xin, Guo Shiming [4] pointed out:At present, the service mode of the library is relatively simple,the training of the literacy and skills is lagging behind, the service mode is compounded, the user experience specialization, the deepening of the skills and the literacy education will become the main trend of the future of the library.Therefore, in order to meet the needs of the user groups, the content of the online lecture system is divided into different levels.

The service tenet of the library must be people-oriented,and the service form is bound to develop in the direction of diversification and hierarchy.Based on the teachers and students in the school and the related scientific research personnel, there are some differences between the different user groups in the demand level of the University library.Although the activities carried out by the library aims to serve the teachers and students as the core, but these activities tend to focus on the basic application, and they can not meet the needs of different user groups hierarchical, and the targeted is not strong, so the effect is often not ideal. Liang Xin, Guo Shiming [4] pointed out:At present, the service mode of the library is relatively simple,the training of the literacy and skills is lagging behind, the service mode is compounded, the user experience specialization, the deepening of the skills and the literacy education will become the main trend of the future of the library.Therefore, in order to meet the needs of the user groups, the content of the online lecture system is divided into different levels.

\subsection{Optimize the time node, improve the lecture efficiency}

The diversity of the library user group determines the time planning of the user group.As the majority of the user groups of university library lectures are teachers and students, the selection of the time node of the library lectures will often integrate various factors, as for the collective interests, overall schedule, avoid conflicts in the time node, creating opportunities for each student to attend the lecture.

The university library online lecture system based on the ASP.NET technology development will effectively break the impact of time node selection. With the help of Internet technology,we can realize the transformation from traditional service to digital library.After user groups loging in the system they can repeat learn a lot of times.This form can effectively avoid the impact of time conflict, at the same time teachers do not need to repeat the teaching.This formcan also reduce the workload of teachers, improving the ratio of input and output.

\subsection{Save space resources and reduce the cost of activities}

The lecture activities in the physical space environment need to occupy the site, audio equipment and so on.In addition to a certain amount of cost, the service life of the device will also produce losses.Under the academic environment of lacking funds for scientific research and advocating the recycling of resources, it is no doubt that it is the best choice to adopt the digital teaching mode.The design and implementation of the online lecture system of university library enables users to study and study under the normal network environment, and will not be restricted by the geographical environment and other factors.

\subsection{Establish evaluation mechanism, optimize service content}

Huwe, University of California Library [5] in the context the construction of digital library, points out that Digital library construction should be combined with big data.Through the library platform to strengthen the collection of user behavior information,then constantly optimize the service level of digital library.Therefore, in order to judging the quality of university library lectures and the results of user groups on the lectures, and we need to use certain indicators or data to measure. The traditional lecture mode generally uses the form of a live examination, and then manually corrected papers. This form not only takes a lot of time, but also having a low efficiency.The online examination function in the online lecture system of the university library can replace this task by replacing it manually. At the same time, the module can also realize the analysis and visualization of the assessment data.

\section{System Design Concept}

\subsection{System design goals}

The design goal of the online lecture system is to start from the actual needs of the user community. Using the help of internet,the system can simplify the lecture process and improve the service quality, it can also rationally utilize the resources, optimize the lectures service, enhance the user experience, change the traditional lecture mode from multiple angles, and build a new digital library online lecture platform.

\subsubsection{Meet the needs of different groups}

Wuhan University Chen Chuanfu [6] in the "large data age digital library construction research,"analyzes the problems faced by digital libraries, he put forward alot of opinions and suggestions about the transformation of ideas, the development of knowledge services, the improvement of financial input mechanism, and the improvement of the quality of librarians. Therefore, the user groups combined with library lectures are complicated. The diversity of the lecture content, the lecture pattern with characteristic of hierarchical, multi type, Diversification can meet the needs of the multi factor conditions. The online lecture system of university library based on the ASP.NET technology will meet the 
diverse needs of the user groups, reducing the time cost of user groups, and enhancing the experience of the user groups.

\subsubsection{Optimize service mode, simplify service flow}

The service object of the library has a variety of disciplines. In order to meet the diverse needs of the user groups, experts and scholars in various fields are invited to participate in the Library Lecture Service.With the help of online lecture system in university library, not only can we get rid of the dependence on the physical space, reduce the economic cost of the library, but also can increase the user groups to obtain knowledge and education opportunities, optimize the experts and user groups all the time, achieve the win-win purpose.

\subsubsection{Establish information feedback mechanism and improve service quality}

The establishment of good communication channels and information feedback mechanism in the network environment is also an important way to enhance the library service.Brition and others with large data technology at Massachusetts Institute of Technology participating in the library service user groups to conduct a deep excavation of the behavior of students and the association analysis[7].After the end of the traditional lecture,there is a short interactive quiz.This step can only grasp the information about the user groups on the content of the lecture, and it also can not quantify the quality of lectures.However,the online lecture system will effectively make up for this deficiency, and achieve the function like SPSS Modeler, Hadoop and so on.The system will be automatically transferred to the online evaluation module after the completion of the learning task of the user group, and then the system will assess the effect of learning of user groups.

\subsection{System design principles}

\subsubsection{Focus on improving service quality}

As the learning resource service center of the University, the library provides the best service for the majority of users.In the era of big data,network academic resources construction developed very quickly.Under such circumstances, the construction of digital library should establish a comprehensive service concept and expand the service mode and knowledge transmission form.Change passive to active, personalized, and new forms of service model will be more welcomed by the user groups.

\subsubsection{Simple and user-friendly system operation}

In the era of big data, the information literacy of the user group is getting higher and higher, and the information service level of the library is also put forward newer and higher requirements.University library lectures online system based on ASP.NET technology will have better man-machine interactive functions and friendly interface. The system function modules in the humanized design reflects the perfect combination with users.In order to meet the increasing needs of the user groups in the future, system structure with flexibility, scalability and the ability of easy to maintain the system provides a basic guarantee for the upgrading and optimization of the latter system.

\subsubsection{Diversified information services}

IFLA stated in the IFLA Digital Library Declaration [8]:University libraries should use their own advantages to provide useful information to users, and use the library's own role in the dissemination of digital media resources.In addition to providing basic online lectures, the diversified information service content makes the service strategy of the university library change in the subtle influence, allowing the system to be recognized by more user groups. Addition to online evaluation function, the system also designed interactive communication, online Q \& A, message boards, comments box and other functional modules.

\section{System Design Flow}

\subsection{Development tools and related technologies}

Online lecture system is built on the platform of ASP.NET and SQL Server database. It runs on the server of the Internet, the user groups accessing by B/S. ASP.NET technology support C\#, Java and other programming languages. It also provides the convenience for the development of the online lecture system, and provides the conditions for the daily management of the system.

SQL Server database as a background database, the system has the advantages that customs application allows the use of Visual Studio development, and make the library with the safety, reliability and high scalability to run the most critical task. The system can reduce the time and cost of development and management data infrastructure, and ensure the security and stability of the system.

\subsection{System structure design}

The online lecture system of university library is a three layer structure, which mainly includes UI layer, business logic layer and data layer. The structure is one of software abstraction hierarchy and a kind of vertical segmentation of complex software. The type of operation is performed at each level in order to segment the code based on its mission to reduce the complexity of the software and improve the maintainability of the system.

ASP.NET works as shown in Figure 1:

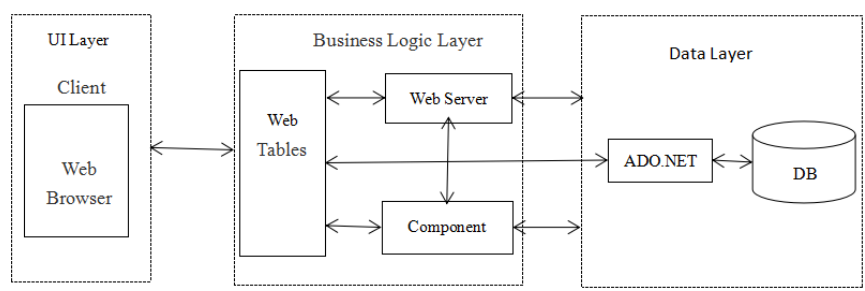

Fig .1. The working principle of ASP.NET 


\subsection{Database Design and Access}

\subsubsection{Database design}

Database is all of the information resources storage warehouse. All the information of users will directly impact on the database. Establishing an efficient and secure database system is an important guarantee of normal operation of the whole system. Using SQL Server database to build a online meeting database, it contains five tables, each table contains the corresponding field,as shown in table 1:

TABLE I. Online meeting database contains tables and fields in the table

\begin{tabular}{cc}
\hline Tables & Fields \\
\hline Administrator & aName, aPwd \\
Teachers & tName, tPwd \\
Users & uName, uPwd \\
Source & ID、Theme、Path \\
Tests & ID、Contents Type、 \\
& Answers Score, GetScore \\
\hline
\end{tabular}

\subsubsection{Database access}

ADO.NET provides consistently access to SQL Server data sources. Data sharing application enables to connect data sources by using ADO.NET to find, update, and modify the internal data of the tables in the online meeting database. ASP.NET provides efficient components to achieve the storage of the database. The introduction of Data Set ADO.NET data set to achieve high-speed data buffer.

Some of the core code for the system to access the database is as follows:

SqlParameter[] pms=new SqlParameter[]

New SqlParameter("@ID",SqlDbType.varchar,50) $\{$ value=txtID.Text.Trim ()$\}$, new SqlParameter("@Pwd",SqlDbType.varchar,50) $\{$ value $=$ txtPwd.Text $\}$; cmd.Parameters.AddRangs(pms);

Con.open(); int $\mathrm{r}=$ cmd.ExecuteNonquery();

\}

\subsection{System function design}

The structure of the online lecture system is shown in Figure 2:

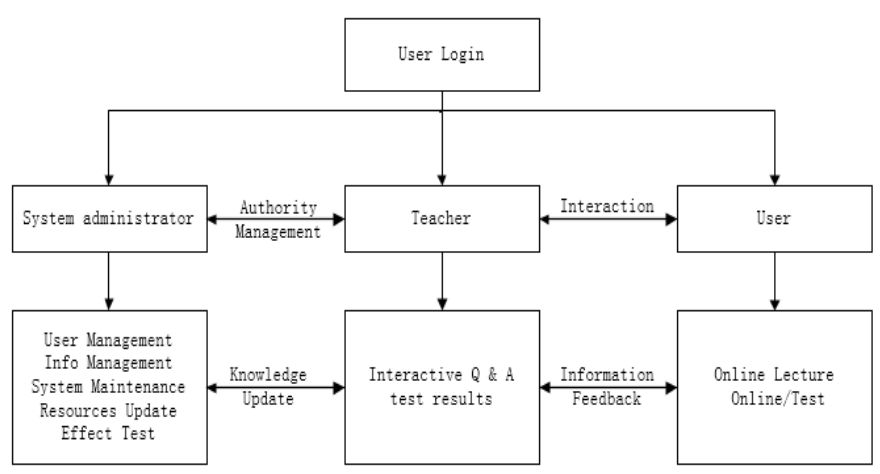

Fig.2. The structure and function of the system

\subsubsection{User information management}

The system administrator has the highest authority, and mainly responsible for user information management, resource update, system maintenance, etc. System administrators make different types of user groups to have a distinct level of authority and within the scope of the exercise of authority. For different types of lectures, the system can update resources in a timely manner. The combination of digital library construction and library service concept to meet the growing user needs of functional groups, optimize system functions, to achieve the continuous upgrading of the system.

\subsubsection{Information resource management}

After the administrator of the various types of user groups to complete the corresponding authorization, the user groups can be within the scope of authority to exercise the appropriate permissions. The teacher will be prepared in advance of teaching resources, examination questions and other information uploaded to the system. When the system is open to the outside world, the user group can go through the system front end, with the user name and login password into the system to learn.At the same time, accessing to the user group can be downloaded in the system corresponding to the courseware resources.

\subsubsection{Online lecture}

After user groups logged into the online lecture system, they can start a targeted learning task. As the learning resources in the system stored for a long time, the user community can avoid the time and space constraints, select the appropriate learning nodes, repeat learning, effective grasp of learning initiative, to avoid the impact of traditional lectures model.The design concept of the system pays more attention to the dissemination and reproduction of knowledge, emphasizes the ability of autonomous learning and training of user groups.

\subsubsection{Lecture evaluation module}

The examination module is the core of the university library online lecture system, and the innovation and uniqueness of the system. The design of the evaluation module combines the needs of the user community and the service concept of the digital library.Through the specific assessment data, the teacher timely and effective grasp of the user group learning situation.In view of the shortcomings in the course of teaching, teachers can adjust the teaching strategies in a timely manner, and constantly optimize the quality of library services.As part of the library to undertake some of the teaching tasks, the user group to master the content of the lectures is the basis for the quality of teacher assessment.

\subsubsection{Interactive quiz module}


The interactive Q \& A module is designed to effectively address short boards present in traditional lecture modes. The survey found that most of the user groups are more inclined to real-time interactive mode of communication. The design of the interactive Q \& A module not only facilitates the user community to ask questions, quickly receives a reply, but also helps the learning and understanding of the course content to a new level for the user groups that are not aware of the problem.At the same time, the interactive Q \& A module design can continue to cultivate the interaction and sharing of user groups.For the problems posed by the user group, the system can be classified analysis, and provide the basis and direction for the library to optimize the service quality.

\subsection{Comparison of the characteristics of 3.5 online lecture system}

\subsubsection{System Design Cost Economy}

System features is the service of the majority of user groups, applications, knowledge sharing.

The biggest feature of the system is the system resources free of charge to the university user groups. The design of the system is simple and simple design style, reducing the time and cost of system development.The system shipped in the campus of the server, to facilitate the daily management of the system, management and upgrading.

\subsubsection{System resource specialization}

The learning resources of the online lecture system are professional and targeted. The learning resources within the system are designed for different subject backgrounds, identity categories and hierarchical needs of the user groups in colleges and universities. At the same time, the content of the teaching is also taught by senior experts in the field, to ensure the professional content of the teaching.In order to improve the learning efficiency, the system can update the learning content and shorten the learning cycle according to the learning situation of the user group.

\subsubsection{Autonomous learning of user groups}

Compared with the traditional face-to-face courses, online lectures system teaching mode can inspire the enthusiasm of the user community to learn independently and cultivate students hands-on and practical ability.Flexible teaching methods are also constantly encourage and guide the user groups from passive learning to active learning changes, to further increase the user group participation, to meet the information access needs and improve the user group integrated information literacy.

\section{Conclusion}

The university library online lecture system is based on the development of ASP.NET technology.The design and implementation of the system not only solve the problems existing in the traditional service model, but also provide the scientific basis for improving the work of the library.However, the system also has some shortcomings. The data obtained from the online evaluation module can be used for shallow analysis, presented in a simple visual form, and shallow data mining.Today, large data theory and technology are increasingly mature, in the enterprise has been widely used.In the future, the system should also introduce the corresponding data mining algorithm to realize the deep analysis and mining of the background data, mastering the development trend of college students' information literacy, giving full play to the data value, providing scientific data for the research of related fields.

\section{REFERENCES}

1. Su Xinning. Opportunities and Challenges for Digital Library in Large Data Times [J]. Journal of Library Science, 2015,06: 4-12.

2. Wu Weici. The development of Chinese library science and the mission of the new generation of library scholars - Report on the establishment of the eighth academic research committee of the China Library Association [J]. Journal of Library Science, 2009, 06: 66-69.

3. Chu Jingli, Wu Dongman. Library development trend research report: environmental analysis and the main strategy [J]. National Library Journal, 2010,01: 3-11.

4. Liang Xin, Guo Shiming. China Mobile Library Service Model Status and Development Trends [J]. Information Work, 2013,05: 98-102.

5. Huwe $\mathrm{T} \mathrm{K}$. Building digital libraries:big data and the library:a natural fit[J]. Computers in Libtaries,2014,34(2):17-18.

6. Chen Chuanfu, Qian Ou, Dai Yuzhu.Study on Digital Library Construction in Large Data Age [J]. Library and Information Work, 2014 (7): 40-45.

7. Renaud J,Brition S,Wang D,et al. Mining library and university data to understand library use patterns[J]. The Electronic Library,2015,33(3):355-372.

8. Zhang Xiaolin. Let the digital library to drive the library service innovation and development: read the "IFLA digital library declaration" [J]. Journal of Library Science, 2010 (3): 73-76. 